\title{
CORRECTION
}

\section{Correction to: Evidence of a gene-environment interaction of NODAL variants and inflammation in preterm birth}

Lisa M. Starr, Taghreed A. Ayash and Daniel Dufort

(c) The Author(s), under exclusive licence to Springer Nature America, Inc. 2021

Journal of Perinatology (2022) 42:1146; https://doi.org/10.1038/s41372-021-01246-9

Correction to: Journal of Perinatology https://doi.org/10.1038/ s41372-018-0073-3, published online 16 February 2018
In this article, the author name Taghreed Heba has changed to Taghreed A. Ayash.

The original article has been corrected. 